\title{
Información sobre I+D y valoración de empresas El caso del sector automotriz en Estados Unidos de Norteamérica y Reino Unido
}

Teresa Duarte Atoche Universidad de Sevilla tduarte@us.es

José Ángel Pérez López Universidad de Sevilla jangel@us.es

\section{José Antonio}

Camúñez Ruiz

Universidad de Sevilla

camunez@us.es

\section{Resumen}

Los Principios de Contabilidad Generalmente Aceptados en EE.UU. requieren del tratamiento como gasto de las inversiones de I+D. Sin embargo, las Normas Internacionales de Información Financiera permiten su capitalización siempre y cuando se cumplan ciertos criterios (IAS 38). En la actualidad, la Junta de Normas Internacionales de Contabilidad (IASB) y el Consejo de Normas de Contabilidad Financiera (FASB) están de acuerdo en unificar esfuerzos para reducir o eliminar las diferencias entre el IFRS y los Principios de Contabilidad Generalmente Aceptados en EE.UU. Los comités del IASB y FASB continúan trabajando sobre las inversiones en I+D; por ello es que en este trabajo se contrastó la relevancia y fiabilidad de la información sobre $\mathrm{I}+\mathrm{D}$ con objeto de poder estimar el valor de mercado de las empresas que cotizan en los mercados de capitales. El método se centró en el empleo en diferentes variaciones del modelo de Ohlson (1995). La muestra estuvo formada por empresas que cotizan en Estados Unidos y en el Reino Unido y que pertenecen al sector del automóvil; el horizonte temporal analizado comprende diez años (1995-2004). Se encontró que los inversores consideran la inversión en I+D con un lapso temporal mayor a un ejercicio económico como un activo; sin embargo, la inversión en I+D corriente es considerada como un gasto del ejercicio sin aportar valor añadido a las compañías; consecuencia de esto, la normativa internacional debería seguir lo regulado por el IASB.

Palabras claves: gastos en I+D, normas internacionales de contabilidad, modelo de Ohlson, valor de mercado. 


\title{
Information about $R \& D$ and companies valuation. The case of the automotive industry in the United States of America and United Kingdom
}

\begin{abstract}
U.S. GAAP require the full expensing of R\&D expenditures. However, International Financial Reporting Standards (IFRS) require capitalization of R\&D cost if it meets certain criteria (IAS 38). At the moment, the International Accounting Standards Board (IASB) and Financial Accounting Standards Board (FASB) agreed to undertake efforts to reduce or eliminate certain narrow differences between IFRS and U.S. GAAP. In regard to R\&D expenditures, the IASB and FASB staff research will continue. This paper aims at examining the relevance and reliability of information about R\&D expenditures, trying to estimate the company value in the Stock Market. Our method consists of different specifications of a valuation model, that is, the Ohlson Model (1995). The sample is composed of U.S. and U.K. listed automotive firms over a 10-year period (1995-2004). Our findings indicate that investors consider that current $\mathrm{R} \& \mathrm{D}$ is an expense and that lagged $\mathrm{R} \& \mathrm{D}$ is an asset. Accordingly, the international regulation should follow IASB standards.
\end{abstract}

Keywords: R\&D, International Accounting Standards, Ohlson Model, Market Value.

\section{Introducción}

Desde hace varias décadas, las diferencias existentes entre la información económico-financiera que presentan las empresas y las necesidades informativas de los usuarios han sido motivo de preocupación e interés creciente en el ámbito académico como lo evidencian los trabajos de autores como Lev y Zarowin (1999), Boulton et al. (2000), Gelb (2002), Shortridge (2004), entre otros.

La mayoría de estos autores sostienen que la causa principal de dicha diferencia es el crecimiento experimentado por los activos intangibles no reconocidos contablemente. Es decir, se produce una relación inversa entre la relevancia de estas inversiones y la utilidad de los sistemas de información contables actuales: a medida que aumenta la importancia de las inversiones en intangibles en la creación de valor añadido para las empresas, se reduce la utilidad de la información económico-financiera divulgada por las mismas.

La numerosa investigación surgida sobre esta cuestión desde la década de los sesenta se ha centrado principalmente en dos aspectos: por un lado, en constatar 
la disminución de interés de los inversores por la información contable ante su pérdida de utilidad (Sougiannis, 1994; Lev y Sougiannis, 1996; Abbody y Lev, 1998; Lev y Zarowin, 1999; Healy et al., 2002; Kothari et al., 2002); por otro, en demostrar la relevancia de las inversiones en I+D que pese a su importancia en la creación de valor añadido en las empresas no eran contablemente reconocidas.

Con respecto al segundo aspecto señalado, son muchos los estudios empíricos que aportan evidencias que avalan esta afirmación; podemos citar, los trabajos de Grabowski y Mueller, 1974; Kamien y Schwartz, 1975; Bublitz y Ettedge, 1989; Johnson y Pazderka, 1993; Chauvin y Hirschey, 1993; Sougiannis, 1994; Lev y Sougiannis 1996 y 1999; Cañibano et al., 2000; Zhao, 2002; Bens et al., 2003; Ballester et al., 2003; Han y Manry, 2004; Callen y Morel, 2005; Mui-Siang y Yeow, 2007; Oswald y Zarowin, 2007; Nguyen et al., 2010, entre otros. No obstante, el contraste empírico de esas evidencias se manifestó como débil y, además, muy pocos estudios han probado la fiabilidad de los valores de las inversiones en I+D (veáse Wyatt, 2008). La falta de evidencia empírica respecto a la fiabilidad de los gastos de I+D (entre los trabajos realizados en torno a esta característica destacamos el realizado por Healy et al., 2002) es el principal problema para su reconocimiento como un activo generador de beneficios futuros. La gran dificultad a la que nos enfrentamos es la incertidumbre que existe en torno al éxito o fracaso de un proyecto de I+D; por tanto, ésta es la faceta del análisis más refutada.

El principal problema para el reconocimiento del I+D era demostrar que estos activos son recursos de beneficios futuros, controlados por la empresa y que se pueden medir con suficiente fiabilidad. Con carácter general, los proyectos de I+D se caracterizan por la incertidumbre que los rodea acerca de su éxito o fracaso. A medida que el proyecto avanza la incertidumbre se reduce y la empresa puede demostrar que generará beneficios en el futuro y que éstos pueden ser medidos con fiabilidad. Ésta es la causa por la que la mayoría de los investigadores y representantes del mundo empresarial defienden la capitalización racional.

La evidencia empírica llamó la atención sobre la necesidad de que los órganos reguladores en el nivel internacional se implicaran en este problema y proporcionaran una solución al deterioro de la utilidad de la información financiera. Captado el interés del Financial Accounting Standard Board (FASB), éste abrió un proceso de consultas y deliberaciones con objeto de incluir esta preocupación en su agenda: quedaba patente la importancia de las inversiones conductoras de la innovación, 
pero los principios de contabilidad generalmente aceptados en el ámbito estadounidense (conocidos por las siglas US GAAP) no permitían su reconocimiento como activos. Paralelamente, el FASB y el International Accounting Standard Board (IASB) comenzaron a trabajar en la armonización de las normas contables relacionadas con este tipo de inversiones como parte del gran proceso de armonización contable a escala internacional que todavía sigue activo.

Un caso que nos permite ilustrar el diferente tratamiento contable internacional de los activos intangibles y las subsecuentes necesidades de armonización sería el relativo a la capitalización del I+D, prohibida en Estados Unidos de manera general (excepto en los desarrollos de software), y la norma europea emitida por el IASB (especialmente la NIC 38) que permite, bajo ciertos requisitos, la capitalización de los costos de desarrollo. De esta manera, se produce un difícil equilibrio entre las dos características más controvertidas de los sistemas contables (relevancia y fiabilidad) que nos induce a considerar que las normas emitidas por el IASB revisten de mayor utilidad a los sistemas contables actuales en el nivel internacional.

Estamos, pues, ante una materia cuyo estudio continúa en la actualidad siendo relevante, y resulta todavía necesario aportar evidencia empírica que ayude a los organismos internacionales sobre la dirección que deberían seguir las normas sobre I+D en el nivel internacional, en aras a una conciliación entre los US GAAP y las NIIF (Normas Internacionales de Contabilidad, emitidas por el IASB) que todavía no se ha producido, pues su discusión ha quedado en suspenso en las agendas de ambos reguladores.

El presente trabajo se enmarca dentro de esta preocupación compartida por los ámbitos académicos y profesionales, por lo que trataremos de contrastar la relevancia y fiabilidad de la información sobre I+D con objeto de poder estimar el valor de mercado de las empresas que cotizan en los mercados de capitales. Ello lo haremos siguiendo la línea de investigación empírica en contabilidad basada en la valoración.

La investigación contable basada en la valoración ha sido ampliamente analizada y utilizada en las últimas décadas (Ver en Barth, 2000; Holthausen y Watts, 2001; Kothari, 2001; Healy y Palepu, 2001); su finalidad es la de relacionar los números contables con una medida del valor de la compañía para evaluar las características de las magnitudes contables y sus relaciones con el valor. Dentro de este mar- 
co de investigación, en nuestro análisis empírico hemos adaptado los modelos de valoración seleccionados para poder relacionar el valor de la compañía con sus características intrínsecas con aquellas que los inversores asumen para valorarla. Concretamente, vamos a emplear como función base el modelo de Ohlson (1995) y a partir del mismo desarrollaremos una serie de funciones de valoración derivadas de sus correspondientes dinámicas de información lineal (DIL).

El desarrollo de esta investigación está estructurado de la siguiente manera: a continuación exponemos la metodología empleada; después analizamos la muestra; posteriormente presentamos los resultados obtenidos del estudio empírico; por último, enumeramos una serie de conclusiones al respecto.

\section{Modelo empírico}

El objetivo general de esta investigación consiste en evaluar si el valor de las inversiones en I+D es relevante; es decir, si los inversores consideran que dicha información es de gran importancia para valorar a las compañías cotizadas en los mercados de valores con el propósito de realizar inversiones rentables.

Utilizaremos un modelo de valoración que relacione el valor de la firma con las características propias que los inversores asumen para valorarla. Según Barth (2000) éste es el factor clave para relacionar el valor de la firma y las cifras contables en un estudio. El modelo de Ohlson (1995), que adopta el planteamiento del modelo de resultado residual para relacionar magnitudes contables con el valor de la empresa, ha sido muy utilizado en la última década porque ha suscitado un gran interés entre los académicos contables. El continuo análisis al que ha sido sometido este modelo (Ohlson, 1995 y 2001; Feltham y Ohlson, 1995 y 1996; Barth y Clinch, 1998; Dechow et al., 1999; Beaver, 1999; Barth et al., 1994 y 2005; Jones, 2000; Callen y Morel, 2001 y 2005; McCrae y Nisson, 2001; Ballester et al., 2003; Han y Manry, 2004; Caro, 2004; Garrod y Valentincic, 2005; Gietzmann e Ireland, 2005; Callen y Segal, 2005; Cazavan-Jeny y Jeanjean, 2005; Giner e Íñiguez, 2006; entre otros) ha permitido su desarrollo y ha demostrado la validez y ventajas de su uso, aunque según la evidencia empírica aportada por distintos académicos (Dechow et al., 1999; Callen y Morel, 2001; Morel, 2003; Caro, 2004; entre otros) presenta una utilidad limitada. 
Este modelo parte de una serie de asunciones ${ }^{1}$ realizadas por Ohlson (1995) que permite explicar el valor de las acciones en función de los fondos propios y de los resultados residuales y de otra información no contenida en las variables anteriores.

Ohlson introduce la dinámica de información lineal que permite vincular valores contables corrientes y futuros, además de definir el comportamiento de cada variable que integra el modelo y sus variantes. Las series evolucionan como un proceso autorregresivo $^{2}$ que indica la forma de cambio de las ganancias anormales esperadas y de la variable "otra información" que constituye el modelo de valoración. Esta última magnitud recoge aquella información no capturada en las ganancias anormales históricas y que modifica la predicción de rentabilidades futuras de la compañía; no obstante, su definición no fue claramente señalada por Ohlson en 1995, aunque hace una propuesta en un trabajo posterior (Ohlson, 2001).

Las dinámicas de información lineal que definen el comportamiento de las ganancias anormales y de otra información son:

$$
\begin{aligned}
& x_{t+1}^{a}=\omega_{11} x_{t}^{a}+Z_{1, t}+\varepsilon_{1, t+1} \\
& Z_{1, t+1}=\gamma_{11} Z_{1, t}+\varepsilon_{2, t+1}
\end{aligned}
$$

Donde $x_{t}^{a}$ son los resultados anormales en el momento $t, Z_{1, t}$ es "otra información" en el momento $t$, mientras que $\omega_{11}$ y $\gamma_{11}$ son los parámetros de persistencia de las ganancias anormales y de la variable de "otra información", respectivamente, cuyos valores no pueden ser menores que cero ni mayores que uno. El límite inferior impuesto a estos coeficientes implica que la persistencia de las ganancias anormales y de "otra información" tiene que ser positiva y el límite superior establece un comportamiento estacionario para las ganancias anormales; es decir, los límites asignados a los parámetros imponen un deterioro en las ganancias anormales causado por las fuerzas de la competencia que motivarán su convergencia a cero con el transcurso del tiempo. Por último, $\varepsilon_{j, t+1}$ es la perturbación estocástica con media cero y no correlacionada con otras variables del modelo.

\footnotetext{
${ }^{1}$ a) El valor de mercado de la firma es igual al valor presente de los dividendos futuros esperados. Su expresión analítica es $P_{t}=\sum_{\tau=1}^{\infty} E_{t}\left[d_{t+\tau}\right](1+r)^{\tau}$

b) Los atributos relacionados con la condición de "excedente limpio" son: i) El cambio del valor contable entre dos periodos es igual a las ganancias menos los dividendos y ii) los dividendos reducen el valor contable corriente, pero no las ganancias corrientes.

c) El comportamiento futuro de la serie temporal de las ganancias anormales y de la variable "otra información", no recogida en las ganancias anormales, se define mediante dinámicas de información lineal.

${ }^{2}$ Esta asunción está basada sobre la intuición de que las ganancias anormales se revertirán debido a la fuerza de la competencia.
} 
El sistema de ecuaciones implica, por un lado, que el valor esperado de las ganancias anormales correspondientes a $t+1$ es igual a las ganancias anormales en el momento $t$, las cuales se consideran persistentes, más "otra información" $\left(Z_{1, t}\right)$. Por otro, la variable $Z_{1, t}$ dependerá exclusivamente de sus valores pasados y no de las ganancias anormales.

Las anteriores ecuaciones — sin necesidad de realizar pronósticos explícitos de dividendos, ni de las ganancias anormales futuras, ni tampoco asunciones adicionales sobre el valor terminal, ${ }^{3}$ como expresa Dechow et al. (1999: 6) — permiten derivar la siguiente función de valoración:

$$
P_{t}=B_{t}+\alpha_{1} x_{t}^{a}+\alpha_{2} Z_{1, t}
$$

Donde $B_{t}$ recoge el valor contable de los recursos propios, $x_{t}^{a}$ representa el valor de los resultados residuales o ganancias anormales, $Z_{1, t}$ representa la variable "otra información", y $\alpha_{1}$ y $\alpha_{2}$ son los coeficientes de las ganancias anormales y de "otra información", respectivamente, definidos a partir de los valores de los parámetros $\omega_{11}$ y $\gamma_{11}$ de las ecuaciones de comportamiento dinámico y de un costo de capital estimado $(r)$. Estos coeficientes están relacionados por las siguientes igualdades:

$$
\begin{gathered}
\alpha_{1}=\frac{\omega_{11}}{1+r-\omega_{11}} \\
\alpha_{2}=\frac{(1+r)}{\left(1+r-\omega_{11}\right)\left(1+r-\gamma_{11}\right)}
\end{gathered}
$$

\section{Modelo empírico de nuestro estudio}

Planteamos el siguiente enfoque para medir la relevancia de la variable I+D. Basándonos en el modelo citado, diseñaremos una serie de dinámicas de información lineal que nos permitirán analizar si los inversores consideran y efectivamente utilizan la información sobre el I+D, que es contabilizada como gasto directamente, para valorar a las compañías.

\footnotetext{
${ }^{3}$ El análisis de los problemas derivados de la necesidad de acotar el horizonte temporal puede verse en Caro (2004).
} 
Resumiendo, nuestro estudio busca arrojar luz al debate y proceso de armonización que en materia de I+D se está produciendo entre el FASB y el IASB. Trataremos de responder si los inversores son capaces de valorar las inversiones de I+D aunque éstas sean tratadas como gasto del ejercicio.

\section{Dinámica de información lineal (I)}

La estimación de la variable "otra información" presenta importantes problemas. Ante la falta de una definición de dicha variable numerosos estudios se han centrado en investigarla. Ohlson (2001), tras las críticas recibidas por esta imprecisión, sugirió que los pronósticos de los analistas podrían ser una aproximación coherente.

Por tanto, Ohlson (2001) define la variable "otra información" como la diferencia entre las expectativas del mercado de las ganancias anormales en el momento $t+1$ (basadas en información contable y no contable) y las expectativas de las ganancias anormales del periodo corriente $t$ (basado en información exclusivamente contable). Consideró que el consenso de los pronósticos de los analistas de las ganancias del próximo año $\left(f_{t}^{t+\tau}\right)$ era una medida razonable de las ganancias esperadas $\left(E_{t}\left[x_{t+\tau}\right]\right)$. Por tanto, si $Z_{i, t}=E_{t}\left[x_{t+\tau}^{a}\right]-\omega_{11} x_{t}^{a}$, siendo $E_{t}\left[x_{t+\tau}^{a}\right]=E_{t}\left[x_{t+\tau}\right]-r B_{t}$, y sustituimos las ganancias esperadas por el consenso de los pronósticos de los analistas, obtenemos que $E_{t}\left[x_{t+\tau}^{a}\right]=f_{t+\tau-1}^{a t+\tau}=f_{t+\tau-1}^{t+\tau}-r B_{t}$. Finalmente, la variable otra información puede ser medida como:

$$
Z_{i, t+\tau}=f_{t+\tau-1}^{a t+\tau}-\omega_{11} x_{t+\tau-1}^{a}=\left(f_{t+\tau-1}^{t+\tau}-r B_{t+\tau-1}\right)-\omega_{11} x_{t+\tau-1}^{a}
$$

Es importante señalar que esta variable permite captar los acontecimientos relevantes que los sistemas contables no recogen, como por ejemplo las inversiones I+D. Por tanto, mediremos los efectos de la magnitud contable I+D sobre las rentabilidades futuras a través de la dinámica de comportamiento de la variable "otra información" $\left(Z_{i, t}\right)$. Es decir, mediante una nueva variante del modelo de Ohlson, en la que definimos la variable $Z_{i, t}$ como los gastos de I+D corrientes, estudiaremos su relevancia. Así, consideramos que el valor de mercado de la compañía viene explicado por el valor neto contable de la misma, por sus ganancias anormales y por las inversiones en I+D que no son capturadas por ninguna de las variables anteriores. Para introducir el I+D en el modelo, asumimos que estos costos afectan al valor de la firma de dos maneras (Callen y Morel, 2005): 
1. Si las inversiones en $\mathrm{I}+\mathrm{D}$ son reconocidas como gasto, éstas afectarán al valor de la empresa negativamente a través de su impacto normal sobre las ganancias corrientes y el valor contable de los fondos propios.

2. Estas inversiones en I+D pueden afectar a los beneficios futuros proporcionando información sobre sus rendimientos futuros en el mercado de capitales. Dichos efectos serían reflejados en las ganancias futuras esperadas a través de la variable "otra información" $\left(Z_{i, t}\right)$.

Resumiendo, en esta versión del modelo de Ohlson introducimos dos modificaciones. Primero asumimos que la variable I+D presenta una estructura autorregresiva; de esta forma introducimos la premisa de que las rentas procedentes de esta variable revertirán por la fuerza de la competencia; es decir que las rentas procedentes de las innovaciones persistirán durante un tiempo, pero como consecuencia de las fuerzas competitivas sufrirán un deterioro que las hará converger a cero. Segundo, siguiendo a Callen y Morel (2005), tomamos los gastos de I+D como la variable $Z_{i, t}$; sin embargo, en nuestro estudio las ganancias anormales se ven afectadas por los gastos de I+D del periodo anterior. Con esta función de valoración pretendemos contrastar la siguiente hipótesis:

$H_{1}$ : El valor del coeficiente de la variable "gastos de $I+D$ " en el momento $t$, registrados como gastos del ejercicio en la función de valoración (DIL I) que ha sido tratado como subrogado de la variable "otra información", será positivo y significativo.

De esta forma, el resultado indicará que el valor de los gastos de I+D son relevantes para la valoración de una compañía, ya que los inversores consideran esta magnitud como generadora de renta futura, a pesar que la norma no permita su capitalización. De todo lo expuesto deducimos la propuesta siguiente de modelización:

Expresión que representa la funcionalidad de la dinámica:

$$
\begin{array}{cc}
x_{t+\tau}^{a}=\omega_{11} x_{t+\tau-1}^{a}+\omega_{12} I D_{t+\tau-1}+\varepsilon_{1, t+\tau} & \tau \geq 1 \\
I D_{t+\tau}=\gamma_{11} I D_{t+\tau-1}+\varepsilon_{2, t+\tau} & \tau \geq 1
\end{array}
$$


Estimaciones de las ganancias anormales bajo las hipótesis habituales sobre las perturbaciones aleatorias:

$$
\begin{array}{cc}
E_{t}\left[x_{t+\tau}^{a}\right]=\omega_{11} x_{t+\tau-1}^{a}+\omega_{12} I D_{t+\tau-1} & \tau \geq 1 \\
E_{t}\left[I D_{t+\tau}\right]=\gamma_{11} I D_{t+\tau-1} & \tau \geq 1
\end{array}
$$

Función de valoración: $\quad P_{t}=B_{t}+\alpha_{1} x_{t}^{a}+\alpha_{2} I D_{t}$

Donde, $\quad \alpha_{1}=\frac{\omega_{11}}{\left(1+r-\omega_{11}\right)}$

$$
\alpha_{2}=\frac{\omega_{12}(1+r)}{\left(1+r-\omega_{11}\right)\left(1+r-\gamma_{11}\right)}
$$

Definición de variables y parámetros:

$x_{t}^{a}$ : Ganancias anormales correspondientes al momento $t$.

$B_{t}$ : Valor contable de los recursos propios en el momento $t$.

$I D_{t}$ : Gastos de $\mathrm{I}+\mathrm{D}$ correspondientes al momento $t$.

$P_{t}$ : Valor de mercado de la firma en el momento $t$.

$r:$ Costos de capital. ${ }^{4}$

$\omega_{11}$ : Parámetro que captura la persistencia de las ganancias anormales. Sus valores deben estar entre 0 y 1 , con objeto de que se cumpla el proceso estacionario de las ganancias anormales.

$\omega_{12}$ : Parámetro que captura el efecto de los gastos de I+D sobre las ganancias futuras.

${ }^{4}$ Existen diversos métodos para estimar el costo de capital. Encontramos desde los modelos más simples, que consisten en considerar que es constante para toda muestra y a lo largo del periodo de análisis, hasta los más complejos, como el modelo CAPM de Sharpe (1964), el de Fama y French (1997), el propio modelo de Ohlson (1995), entre otros. El objetivo de este estudio no es evaluar los métodos alternativos para estimar el costo de capital; por ello, y teniendo en cuenta que no es una variable que altere la esencia de los resultados, hemos considerado que la tasa de descuento fluctúa de un periodo a otro, pero es constante para todas las compañías que

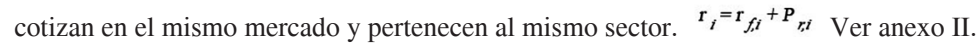


$\gamma_{11}$ : Parámetro de persistencia de los costos de I+D ${ }^{5}$. Sus valores deben estar comprendidos entre 0 y 1 . De esta forma se cumple la asunción de que el I+D sigue un proceso estacionario.

$\alpha_{1}$ : Coeficiente de la variable "ganancias anormales" en la función de valoración.

$\alpha_{2}$ : Coeficiente de la variable I+D en la función de valoración. Si este coeficiente es positivo, nos indicará que los inversores valoran positivamente las inversiones de $\mathrm{I}+\mathrm{D}$, considerándolas generadoras de rentas futuras.

\section{Dinámica de información lineal (II)}

Este modelo es una variante de la DIL (I). Las premisas que condicionan esta dinámica de información son los siguientes: a) los costos de I+D los consideramos estacionarios; es decir, su comportamiento sigue un proceso autorregresivo de orden 1 (AR1) dada la sencillez y proximidad a lo real de estos procesos; b) siguiendo a Callen y Morel (2005), tomamos los gastos de I+D, del ejercicio corriente y del ejercicio anterior, como la variable $Z_{1, t}$, y las ganancias anormales son afectadas por los gastos de I+D del ejercicio corriente y por aquellos correspondientes a un periodo inmediatamente anterior. Por tanto, hemos introducido el valor del I+D con un retardo, ya que entendemos que el efecto positivo de estas inversiones sobre el valor de una empresa puede producirse un año después de su desembolso. Parece lógico pensar que los inversores necesitan analizar la evolución del proyecto de I+D durante un periodo amplio para conocer la viabilidad del mismo con objeto de considerarlo gasto o inversión.

Con esta función de valoración contrastaremos la hipótesis $\mathrm{H}_{2}$ : El valor del coeficiente de la variable "gastos de $I+D$ " en el momento $t-1$ en la función de valoración (DIL II) es positivo y significativo. Los resultados pondrán de manifiesto si los inversores consideran relevante el gasto en I+D del ejercicio corriente y el del año anterior; es decir, si el efecto del I+D sobre los resultados de la compañía se extiende al año corriente y al siguiente.

\footnotetext{
${ }^{5}$ Consideramos que en la actualidad y para las empresas intensivas en $\mathrm{I}+\mathrm{D}$, como es el sector del automóvil y partes del mismo, la inversión en I+D es un factor clave para la creación y mantenimiento de ventajas competitivas. En efecto, las empresas pertenecientes a estos sectores presentan departamentos de I+D cuyos presupuestos suponen inversiones muy importantes y cuya vigencia no se pone en duda.
} 
La modelización que subyace en este contexto es la siguiente:

Expresión que representa la funcionalidad de la dinámica:

$$
\begin{array}{cc}
x_{t+\tau}^{a}=\omega_{11} x_{t+\tau-1}^{a}+\omega_{12} I D_{t+\tau-1}+\omega_{13} I D_{t+\tau-2}+\varepsilon_{1, t+\tau} & \tau \geq 1 \\
I D_{t+\tau}=\gamma_{11} I D_{t+\tau-1}+\varepsilon_{2, t+\tau} & \tau \geq 1
\end{array}
$$

Estimaciones de las ganancias anormales, tomando valor esperado y usando las hipótesis habituales sobre las perturbaciones aleatorias:

$$
\begin{array}{cc}
E_{t}\left[x_{t+\tau}^{a}\right]=\omega_{11} x_{t+\tau-1}^{a}+\omega_{12} I D_{t+\tau-1}+\omega_{13} I D_{t+\tau-2} & \tau \geq 1 \\
E_{t}\left[I D_{t+\tau}\right]=\gamma_{11} I D_{t+\tau-1} & \tau \geq 1
\end{array}
$$

Función de valoración:

$$
P_{t}=B_{t}+\alpha_{1} x_{t}^{a}+\alpha_{2} I D_{t}+\alpha_{3} I D_{t-1}
$$

Donde, $\quad \alpha_{1}=\frac{\omega_{11}}{\left(1+r-\omega_{11}\right)}$

$$
\begin{aligned}
& \alpha_{2}=\frac{\omega_{12}(1+r)+\omega_{13}}{\left(1+r-\omega_{11}\right)\left(1+r-\gamma_{11}\right)} \\
& \alpha_{3}=\frac{\omega_{13}}{\left(1+r-\omega_{11}\right)}
\end{aligned}
$$

Definición de variables y parámetros:

$x_{t}^{a}$ : Ganancias anormales correspondientes al momento $t$.

$B_{t}$ : Valor contable de los recursos propios en el momento $t$

$I D_{t}$ : Gastos de $\mathrm{I}+\mathrm{D}$ correspondientes al momento $t$.

$I D_{t-1}$ : Gastos de I+D correspondientes al momento $t-1$. 
$P_{t}$ : Valor de mercado de la firma en el momento $t$.

$r$ : Costo de capital.

$\omega_{11}$ : Parámetro que captura la persistencia de las ganancias anormales. Sus valores deben estar entre 0 y 1 , con objeto de que se cumpla el proceso estacionario de las ganancias anormales.

$\gamma_{11}$ : Parámetro de persistencia de los costos de I+D. Sus valores deben estar comprendidos entre 0 y 1 , de esta forma se cumple la asunción de que el I+D sigue un proceso estacionario.

$\alpha_{1}$ : Coeficiente de la variable "ganancias anormales" en la función de valoración.

$\omega_{12}$ : Parámetro que captura el efecto de los gastos de I+D, en el momento t, sobre las ganancias futuras.

$\omega_{13}$ : Parámetro que captura el efecto de los gastos de $\mathrm{I}+\mathrm{D}$, incurridos en el momento $\mathrm{t}-1$, sobre las ganancias futuras.

$\alpha_{2}$ : Coeficiente de la variable $I D_{t}$ en la función de valoración.

$\alpha_{3}$ : Coeficiente de la variable $I D_{t-1}$ en la función de valoración.

\section{Muestra}

La muestra objeto de nuestro estudio la constituye empresas del sector del automóvil y de componentes que cotizan en los mercados de capitales de Estados Unidos y del Reino Unido. Elegimos el sector del automóvil y sus componentes por tratarse tradicionalmente de grandes inversores en I+D. Además, seleccionamos los mercados de capitales de Estados Unidos y el Reino Unido por ser los más desarrollados en el nivel internacional y donde mayor número de empresas, tanto nativas como extranjeras, cotizan. Asimismo, en dichos mercados las decisiones tomadas por los órganos reguladores contables en el nivel internacional más importantes, FASB e IASB, tienen mayores implicaciones directas.

Los datos empleados en el estudio empírico han sido obtenidos de varias fuentes de información. Los relacionados con los precios de cotización de las acciones han sido obtenidos de la base DATASTREAM. Los datos contables históricos, 
tales como las ganancias básicas por acción, el valor neto contable de los fondos propios, el número de acciones, el activo total y los gastos en investigación y desarrollo incurridos por las compañías, han sido recabados de las bases de datos Thomson One Banker, LexisNexis y de los mismos estados financieros divulgados por las propias compañías en sus páginas web ${ }^{6}$ y en la página de la $\mathrm{SEC}^{7}$ para las empresas cotizadas en Estados Unidos.

La muestra total puede ser dividida claramente en dos submuestras: i) una formada por las empresas que cotizan en los mercados de capitales de Estados Unidos, que están obligadas a registrar como gasto del ejercicio todos los gastos de I+D y ii) otra formada por las empresas que cotizan en el Reino Unido, que deben registrar como gasto del ejercicio todos los desembolsos correspondientes a la investigación, aunque pueden elegir entre capitalizar o no aquellos gastos de desarrollo que cumplan con los requisitos exigidos por la norma.

El periodo que abarca nuestro estudio comprende diez años, desde 1995 hasta 2004. Hemos acotado el horizonte temporal en 2004 porque a partir de 2005 las empresas que cotizan en los mercados de capitales de la Unión Europea, y que presenten estados financieros consolidados, están obligadas a elaborar y divulgar sus estados financieros según las NIC/NIIF. Este cambio normativo provocaría una distorsión en la información financiera de las compañías que forman la muestra desvirtuando los resultados del estudio empírico.

La muestra total está formada por 116 empresas, de las que 96 cotizan en los mercados de Estados Unidos y 20 en los del Reino Unido durante un periodo de diez años. Así, el número total de observaciones asciende a 1 160. Aunque, con la eliminación de las observaciones que distorsionaban el estudio, la muestra se ha visto reducida desde un $17 \%$ en 1997 hasta un 59\% aproximadamente en $2001 .^{8}$

Las variables empleadas están deflactadas en términos por acción, de igual forma que hicieron Dechow et al. (1999), McCrae y Nilsson (2001), Zhao (2002), Callen y Morel (2005), Cazavan-Jeny y Jeanjean (2006), entre otros. Según Ohlson (2001), este método puede transgredir la condición de excedente limpio subya-

\footnotetext{
${ }^{6}$ http://www.carol.co.uk/search.php

${ }^{7}$ http://www.sec.gov/edgar/searchedgar/companysearch.html

${ }^{8}$ Véase en el anexo I el análisis estadístico de la muestra.
} 
cente en el modelo de renta residual si el número de acciones en circulación y manos de los inversores cambia de forma importante. Pero como afirman McCrae y Nilsson (2001) el uso de magnitudes totales también introduce problemas con las posibles contribuciones o distribuciones de capital no apreciadas en los valores de mercado. Para eliminar los problemas de escala que pudieran surgir, hemos deflactado las variables del modelo mediante el activo total por acción, ya que consideramos que esta variable representa adecuadamente el tamaño o escala de las compañías que forman la muestra, proporcionándonos variables que mitigan el problema de heteroscedasticidad, al igual que hicieron Caro (2004), Aboody et al. (2005), entre otros. Posteriormente, y si fuera necesario, utilizaremos el test de errores estándar de White (1980) para detectar la presencia de heteroscedasticidad en los errores de las regresiones ajustadas y en aquellos casos donde no logremos eliminar los problemas de heteroscedasticidad procederemos a eliminar las observaciones extremas que provoquen dichos efectos.

\section{Resultados del estudio empírico}

Los resultados obtenidos del contraste empírico de la primera función de valoración son presentados en el cuadro 1 .

En la DIL I asumimos que los costos de I+D afectan al valor de la firma de dos formas (Callen y Morel, 2005): por un lado, las inversiones en I+D son reconocidas como gasto, por lo que éstas afectarán al valor de la empresa negativamente a través de su impacto normal sobre las ganancias corrientes y el valor contable de los fondos propios; por otro lado, dichas inversiones pueden afectar a los beneficios futuros, proporcionando información sobre sus rendimientos futuros en el mercado de capitales. Estos efectos serían reflejados en las ganancias futuras esperadas a través de la variable "otra información" $\left(Z_{i, t}\right)$. 


\section{Cuadro 1 \\ Resultados de la función de valoración I}

\begin{tabular}{c|c|c|c|c}
\hline \multicolumn{5}{c}{ Función de valoración I. $P_{t}=B_{t}+\alpha_{1} x_{t}^{a}+\alpha_{2} I_{t}$} \\
\hline \multirow{2}{*}{ Años } & $\alpha_{1}$ & & $R^{2}$ & Test White : \\
& & $\alpha_{2}$ & $n R^{2}\left(P^{- \text {valor }}\right)$ \\
\hline 1995 & $14.7859 * *$ & -0.0137 & 0.9921 & $8.1378(0.0870)$ \\
\hline 1996 & $4.7979 *$ & $-0.0061 * *$ & 0.2477 & $10.3189(0.0666)$ \\
\hline 1997 & $4.1469 * *$ & 0.0253 & 0.2769 & $4.0138(0.5474)$ \\
\hline 1998 & $3.0739 * *$ & $-1.0243 *$ & 0.6598 & $4.5236(0.4767)$ \\
\hline 1999 & $6.1009 * *$ & $-0.0020 * *$ & 0.2512 & $3.2746(0.6577)$ \\
\hline 2000 & $6.8280 * *$ & $-0.0039 * *$ & 0.6579 & $5.2722(0.3835)$ \\
\hline 2001 & $6.0664 * *$ & 0.0005 & 0.6145 & $2.3607(0.7973)$ \\
\hline 2002 & $5.3973 * *$ & $0.0010 * *$ & 0.6150 & $9.7902(0.0557)$ \\
\hline 2003 & $9.0484 * *$ & -0.0150 & 0.8667 & $7.1849(0.2072)$ \\
\hline 2004 & $11.6013 * *$ & -0.0082 & 0.8048 & $6.2657(0.2812)$ \\
\hline$* *$ Significativo al 5\%. & & & \\
\hline Significativo al $10 \%$. & & &
\end{tabular}

La hipótesis nula esbozada y a contrastar con la función de valoración que se deriva de esta dinámica consiste en que $H_{1}$ : los coeficientes de la variable $I+D$ -tratada en regresión como "otra información" - serán positivos y significativos para la ventana temporal seleccionada.

Como podemos observar en el cuadro 1 y más resumidamente en el cuadro 2, donde se muestra la media y mediana de los años analizados, las ganancias anormales son relevantes y están relacionadas positivamente con el precio de las acciones de las compañías. Por tanto, en la explicación del valor de mercado de las compañías, las ganancias anormales presentan un peso medio (7.1847, ver cuadro 2 ) substancial y significativo.

Por su parte, los coeficientes de los gastos de I+D son significativos para el 50\% de los años que forman el horizonte temporal analizado; el valor de su media y mediana son negativos. Ello implica que las inversiones en I+D realizadas por las empresas del sector automotriz y de autopartes, las cuales forman parte de nuestra muestra, son consideradas por los inversores como gastos del ejercicio corriente, ya que su relación con el precio es negativa. Estos resultados que coinciden con 
los obtenidos por Cazavan-Jeny y Jeanjean (2006) y Callen y Morel (2005) y que no verifican la primera hipótesis esbozada, en contra de los resultados obtenidos por la mayoría de la literatura previa como Sougiannis (1994), Green et al. (1996), Aboody y Lev (1998), Healy et al. (2001), Han y Manry (2004), entre otros.

\section{Cuadro 2}

Resumen de los resultados de la función de valoración I

\begin{tabular}{|c|c|c|c|c|c|}
\hline \multicolumn{6}{|c|}{ Función de valoración I. $P_{t}=B_{t}+\alpha_{1} x_{t}^{a}+\alpha_{2} I D_{t}$} \\
\hline & $\alpha_{1}$ & $\alpha_{2}$ & $R^{2}$ & Test White: & $n R^{2}\left(P^{- \text {valor }}\right)$ \\
\hline Media & $7.1847 * *$ & $-0.2070 * *$ & 0.5987 & \multicolumn{2}{|c|}{$6.1142(0.3560)$} \\
\hline Mediana & $6.0836 * *$ & $-0.0039 * *$ & 0.6365 & \multicolumn{2}{|c|}{$5.7690(0.3323)$} \\
\hline
\end{tabular}

El test de white mantiene la existencia de homoscedasticidad en todas las muestras utilizadas para contratar esta función de valoración a lo largo de la ventana temporal.

En resumen, los resultados obtenidos indican que las inversiones corrientes en I+D no son consideradas como generadoras de renta futuras por los inversores; éstos consideran necesario que se pruebe la fiabilidad del proyecto de investigación y desarrollo. Por ello, seguimos el análisis e introducimos una segunda variable formada por la inversión en I+D con un año de retardo. Con esta variable aceptaremos o no la hipótesis de que los inversores necesitan conocer la viabilidad del proyecto de investigación para considerarlo relevante y positivamente relacionado con el valor de la compañía.

En la función de valoración de la DIL II introducimos una nueva variable, $I D_{t-1}$, que refleja el valor del I+D del ejercicio anterior, con la que contrastamos si los inversores necesitan un periodo de un año para discernir si el proyecto de investigación en el que está involucrada la compañía será o no viable; es decir, si generará beneficios en el futuro. La segunda hipótesis nula a contrastar establece que $\mathrm{H}_{2}$ : los gastos de $I+D$ con un periodo de retardo serán considerados relevantes por los inversores y su relación con el valor de mercado será positiva. 


\section{Cuadro 3}

\section{Resultados de la función de valoración II}

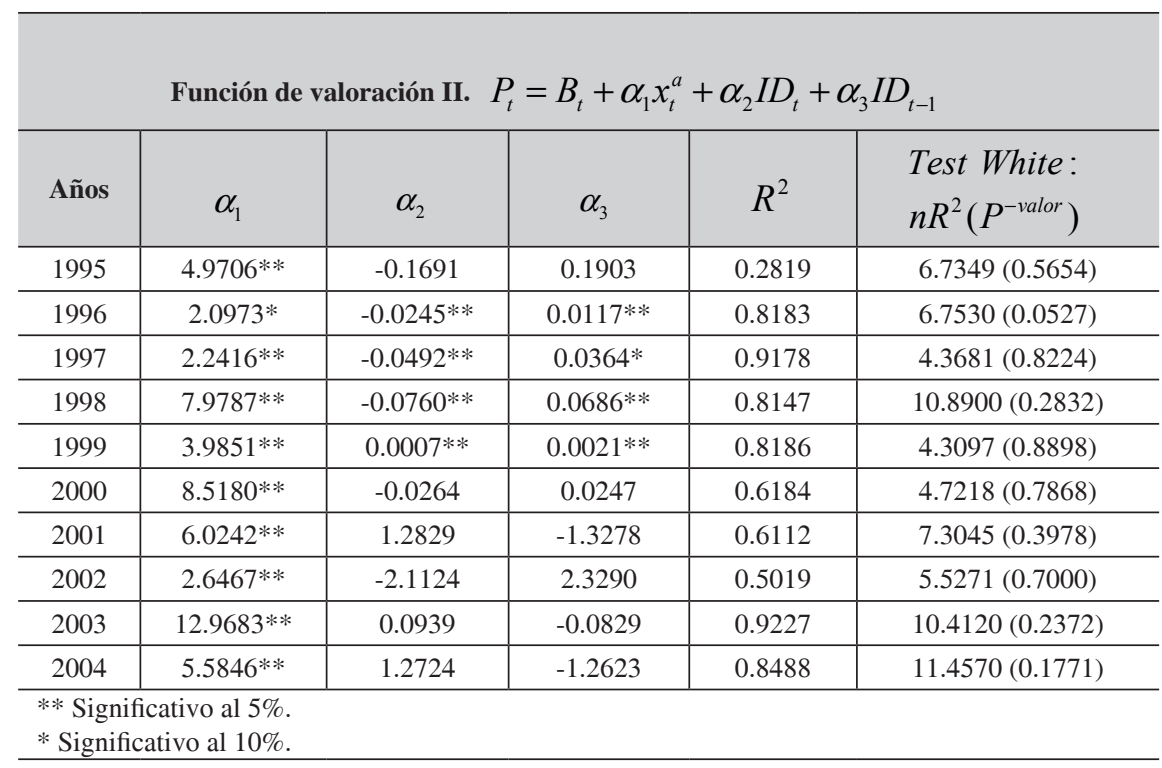

Los resultados demuestran que los coeficientes de las ganancias anormales, una vez más, son positivos y significativos a lo largo de la ventana temporal analizada, aunque su peso medio se ha visto reducido en dos puntos, como se observa en el cuadro 3.

Por su parte, los coeficientes de los gastos de I+D del ejercicio corriente son significativos en un $40 \%$ de la muestra. Su valor medio es negativo, coincidiendo con los resultados obtenidos en la función de valoración de la DIL I, aunque su peso relativo en la función se ha visto reducido debido a la introducción de la nueva variable.

Los coeficientes estimados para el I+D del ejercicio anterior indican que están positivamente relacionados con el valor de la compañía en un $70 \%$ de los años, aunque son significativos en un $40 \%$ de la muestra total y son negativos en un $30 \%$ de los años, aunque no significativos. El valor medio de los coeficientes significativos es positivo, lo que indica que los inversores necesitan un periodo mínimo de un ejercicio económico para estimar la viabilidad del proyecto de investigación y una vez transcurrido dicho lapso consideran que el I+D es una inversión. Así, los resultados verifican la segunda hipótesis. 
El contraste verifica la segunda hipótesis en un $40 \%$ de la muestra total, que supera significativamente el 25\% que obtuvieron Callen y Morel (2005) con un estudio similar al nuestro. La diferencia entre ambos estudios estriba en el tratamiento de la variable I+D: mientras nosotros hemos establecido un comportamiento autorregresivo para esta variable, Callen y Morel lo describieron como aleatorio, como ya se ha comentado. La hipótesis de autorregresión de orden 1 es realista (el pasado inmediato influye más que el pasado alejado en el tiempo sobre el resultado actual) y sencillo. Por tanto, a la luz de los resultados, creemos que nuestro enfoque es más cercano a la realidad que el establecido por estos autores.

\section{Cuadro 4}

\section{Resumen de los resultados de la función de valoración II}

\begin{tabular}{|c|c|c|c|c|c|}
\hline \multicolumn{6}{|c|}{ Función de valoración II. $P_{t}=B_{t}+\alpha_{1} x_{t}^{a}+\alpha_{2} I D_{t}+\alpha_{3} I D_{t-1}$} \\
\hline & $\alpha_{1}$ & $\alpha_{2}(40 \%)$ & $\alpha_{3}(40 \%)$ & $R^{2}$ & $\begin{array}{l}\text { Test White: } \\
n R^{2}\left(P^{-v a l o r}\right)\end{array}$ \\
\hline Media & $5.7015^{* *}$ & $-0.0372 * *$ & $0.0297 * *$ & 0.7154 & $7.2480(0.4912)$ \\
\hline Mediana & $5.2776 * *$ & $-0.0368 * *$ & $0.0240 * *$ & 0.8165 & $6.7440(0.4816)$ \\
\hline
\end{tabular}

Lo anteriormente expuesto puede observarse claramente en el cuadro 4, donde se muestra la media y mediana de los años analizados que resumen los resultados de la muestra completa. En ella incluimos la media y mediana de los coeficientes estimados y significativos que para la variable $I D_{t}$ e $I D_{t-1}$ suponen un $40 \%$ de la muestra total. Esto nos lleva a rechazar la $H_{1}$ y a aceptar la $H_{2}$.

\section{Conclusiones}

En este estudio hemos analizado la relevancia de la información sobre I+D mediante la estimación del valor de mercado de una muestra de compañías del sector del automóvil y componentes del mismo que cotizan en Estados Unidos y el Reino Unido. Nuestro objetivo es arrojar luz al actual debate que se está produciendo en el seno de las dos comisiones reguladoras más importantes en el nivel internacional, el FASB y el IASB, en torno al tratamiento del I+D. 
Los resultados de nuestro trabajo aportan importantes evidencias sobre el camino que debería seguir la armonización contable en materia de I+D con objeto de que el sistema de información económico-financiera se revista de la relevancia necesaria para los inversores y los usuarios externos en general. Resumidamente, presentamos las conclusiones extraídas del estudio.

La información presentada indica que los inversores de las compañías que pertenecen al sector del automóvil o componentes del mismo, y que cotizan en una de las dos bolsas de valores más importantes del mundo, consideran que el gasto en I+D corriente no es inversión y que necesitan un lapso temporal de un ejercicio económico, como mínimo, para estimar razonablemente si dichos desembolsos generarán beneficios en el futuro. Esto último implica que el gasto de I+D que se desembolsó durante el ejercicio anterior es considerado como inversión para estimar el valor de mercado de la compañía en el año corriente.

Nuestros resultados, por tanto, abogan por un cambio en las normas contables internacionales en materia de I+D que exijan la capitalización de los gastos de I+D una vez se haya testado la viabilidad del proyecto. En este sentido, la armonización contable sobre I+D debería seguir la dirección tomada por el IASB. En otras palabras, la conciliación de las diferencias señaladas tendría que pasar por un acercamiento del SFAC No 2 a la NIC 38, con lo que se lograría revestir de mayor utilidad al único sistema de información contable en el nivel internacional que existirá en el futuro.

Este trabajo no está exento de limitaciones. La primera consiste en que el modelo que desarrollamos utiliza datos para una sola industria. Aunque la industria automovilística proporciona un ajuste ideal para estudiar los efectos de la contabilidad de $\mathrm{I}+\mathrm{D}$, nuestros resultados no deben generalizar a otras industrias. Segundo, hemos asumido que todas las firmas tienen capacidades similares para el desarrollo de tecnología que no cambia en un cierto tiempo; por tanto, existe la posibilidad de que nuestros resultados sean sensibles a la omisión del riesgo. Todo ello será objeto de mejoras en futuras investigaciones. Concretamente sería interesante analizar la relación entre los gastos de I+D y los rendimientos de las compañías; es decir, utilizando un modelo de rentabilidades que, como establece Easton (1999) y Aboody y Lev (1998), controla los problemas de correlación y mitiga el efecto de variables omitidas; de esta forma podríamos obtener estimaciones más robustas. Asimismo, sería interesante ampliar la muestra a sectores más y menos intensivos en I+D que el sector del automóvil y componentes. 


\section{Referencias}

Aboody, D., J. Hughes y Liu Jing (2005). Earnings Quality, Insider Trading, and Cost of Capital. Journal of Accounting Research 43(5): 651-673.

y B. Lev (1998). The Value Relevance of Intangibles: The Case of Software Capitalization. Journal of Accounting Research 36: 161-191.

(2000). Information Asymmetry, I+D, and Insider Gains. The Journal of Finance LV(6): 2747-2766.

(2001). I+D Productivity in the Chemical Industry. Working paper. New York.

Amir, E. y B. Lev (1996). Value-relevance of nonfinancial information: The wireless communications industry. Journal of Accounting and Economics 22: 3-30.

y T. Sougiannis (2003). Do financial analysts get intangibles? European Accounting Review 12(4): 635-659.

Ballester, M., M. García-Ayuso y J. Livnat (2003). The Economic Value of the I+D Intangible Asset. European Accounting Review 12(4): 605-633.

Barth, M. E. (1994). Fair Value Accounting: Evidence from Investment Securities and the Market Valuations of Banks. The Accounting Review 69(1): 1-25.

y S. Kallapur (1996). The effects of cross-sectional scale differences on regression results in empirical accounting research. Contemporary Accounting Research 13: 527-567.

y G. Clinch (1998). Revalued financial, tangible and intangible assets: associations with share prices and non-market-based value Estimates. Journal of Accounting Research Supplement 36: 199-233.

(2000). Valuation-based accounting research: Implications for financial reporting and opportunities for future research. Accounting and Finance 40: 7-31. 
, W.H. Beaver y W.R. Landsman (2001). The relevance of the value relevance literature for financial accounting standard setting: Another view. Journal of Accounting and Economics 31: 77-104.

(2005). Accruals, Accounting-Based Valuation Models, and the Prediction of Equity Values. Journal of Accounting, Auditing and Finance 20(4): 311-345.

Beaver, W. (1999). Comments on 'An empirical assessment of the residual income valuation model'. Journal of Accounting and Economics 26: 35-42.

Bens, D. A., J.D. Hanna y X.F. Zhang (2003): Research and Development, Risk, and Stock Returns. Working paper, March.

Boulton, R. y B. Libert (2000). A Business Model for a New Economy. Journal of Business Strategy 21(4): 29-36.

Bublitz, B. y M. Ettedge (1989). The Information in Discretionary Outlays: Advertising, Research, and Development. The Accounting Review LXIV(1): 108-124.

Cabedo, J.D. y J.M. Tirado (2007). El beneficio anormal en el modelo de Ohlson: Una propuesta para su estimación. Revista de Contabilidad 10(1): 75-98.

Callen, J. y M. Morel (2001). Linear Accounting Valuation When Abnormal Earnings Are AR (2). Review of Quantitative Finance and Accounting 16(3): 191-203.

(2005). The valuation relevance of I+D expenditures: Time series evidence. International Review of financial Analysis 14: 304-325.

Callen, J. y D. Segal (2005). Empirical Tests of the Feltham-Ohlson (1995) Model. Review of Accounting Studies 10: 409-429.

Cañibano, L., M. García-Ayuso y P. Sánchez (2000). La valoración de los intangibles: Estudios de innovación vs información contable-financiera. Análisis Financiero 80: 6-24. 
Caro Fernández, S. (2004). Los modelos de valoración basados en magnitudes contables: Análisis teórico y evaluación empírica de diferentes dinámicas de información lineal. Tesis Doctoral, Universidad de Sevilla.

Cazavan-Jeny, A. y T. Jeanjean (2006). The Negative Impact of I+D Capitalization: A value Relevance Approach. European Accounting Review 15(1): 37-61.

Chan, L., J. Lakonishok y T. Sougiannis (2001). The Stock Market Valuation of Research and Development Expenditures. The Journal of Finance 56(6): 2431-2459.

Chan, S.H., J.D. Martin y J.W. Kensinger (1990). Corporate Research and Development expenditures and Share Value. Journal of Financial Economics 26: 255-276.

Chauvin, D.W. y M. Hirschey (1993). Advertising, I+D Expenditures and the Market Value of the Firm. Financial Management 22(4): 128-140.

Chiang, C.C. y Y.M. Mensah (2004). The Determinants of Investor Valuation of I+D Expenditure in the Software Industry. Review of Quantitative Finance and Accounting 22: 293-313.

Chung, K. H., P. Wright y B. Kedia (2003). Corporate Governance and Market Valuation of Capital and R\&D Investments. Review of Financial Economic 12: 161-172.

Cohen, W. M. y D.A. Levinthal (1989). Innovation and Learning: The two Faces of I+D. The Economic Journal 99: 569-596.

Dechow, P., A. Hutton y R. Sloan (1999). An empirical assessment of the residual income valuation model. Journal of Accounting and Economics 26: 1-34.

Doukas, J. y L. Switzer (1992). The stock market's view of R\&D spending and market concentration. Journal of Economic and Business 23: 191-216.

Ely, K. y G. Waymire (1999): Intangibles Assets and Stock Prices in the Pre-SEC Era. Journal of Accounting Research 37: 17-51. 
Feltham, G. y J. Ohlson (1995). Valuation and Clean Surplus Accounting for Operating and Financial Activities. Contemporary Accounting Research 11-2: 689-731.

(1996). Uncertainty Resolution and the Theory of Depreciation Measurement. Journal of Accounting Research 34 (2): 209-234.

Financial Accounting Standards Board (1974). Statement of Financial Accounting Standards, N 2, Accounting for Research and Development Costs. FASB, Norwalk.

(1985). Statement $N^{\circ} 86$. Accounting for the Costs of Computer Software to Be Sold, Leased, or Otherwise Marketed. Norwalk, Connecticut.

(1999). The IASC-US Comparison Project: A Report on the Similarities and Differences between IASC Standards and US GAAP-Second Edition. Norwalk, Connecticut.

Francis J. y K. Schipper (1999). Have Financial Statements lost Their Relevance? Journal of Accounting Research 37 (2): 319-352.

Garrod, N. y A. Valentincic (2005): The Term Structure of Implicit Discount Rates in Security Valuation. Journal of Business Finance \& Accounting 32 (7-8): 1237-1274.

Gelb, D. (2002). Intangible Assets and Firms' Disclosures: An Empirical Investigation. Journal of Business Finance \& Accounting 29 (3-4): 457-476.

Gietzmann, M. y J. Ireland (2005). Cost of Capital, Strategic Disclosures and Accounting choice. Journal of Business Finance \& Accounting 32 (3-4): 599-641.

Giner Inchausti, B. y R. Íñiguez Sánchez (2006). La capacidad de los modelos Feltham-Ohlson para predecir el resultado anormal: Una aplicación empírica. Revista Española de Financiación y Contabilidad XXXV(132): 729-759.

Grabowski, H.G. y D.C. Mueller (1974). Rates of Return on Corporate Investment, Research and Development and Advertising. Working Paper. Cornell University. 
(1978). Industrial Research and Development, intangible capital stocks, and firm profit rates. The Bell Journal of Economics 9 (2): 328-343.

Green, J. P., A.W. Stark y M. Hardy (1996). UK Evidence on the Market Valuation of Research and Development Expenditure. Journal of Business Finance and Accounting 23 (2): 191-216.

Han, B. y D. Manry (2004). The value-relevance of I+D and advertising expenditures: Evidence from Korea. The International Journal of Accounting 39: 155-173.

Healy, P., S. Myers y C. Howe (2002). R\&D Accounting and the trade off between Relevance and Objectivity. Journal of Accounting Research 40: 677-710.

Hirschey, M. (1982). Intangible Capital Aspects of Advertising and I+D Expenditures. The Journal of Industrial Economic 4: 375-390.

Hirschey, M. y J.J. Weygandt (1985). Amortization Policy for advertising and Research and Development expenditures. Journal of Accounting Research 23 (1): 326-335.

Johnson, L. D. y B. Pazderka (1993). Firm Value and Investment in I+D. Managerial and Decision Economics 14: 14-24.

Jones, D. (2000): The relative importance of earnings and other information in the valuation of $\mathrm{I}+\mathrm{D}$ intensive firms. PhD. University of Colorado.

Kamien, M. y N. Schwartz (1975): Market Structure and Innovation: A Survey. Journal of Economic Literature March: 1-37.

Kothari, S.P., T.E. Laguerre y J.L. Andrew (2002). Capitalization versus Expensing: Evidence on the Uncertainty of Future Earnings from Capital Expenditures Versus I+D Outlays. Review of Accounting Studies 7 (4): 355-382.

Larrán Jorge, M. y J.M. Piñero López (2005): El modelo de Ohlson (1995): ¿Hemos llegado realmente a comprenderlo? Revista de Contabilidad 8 (16): 115-149. 
Lev, B. y T. Sougiannis (1996). The capitalization, amortization, and value-relevance of I+D. Journal of Accounting and Economics 21: 107-138.

(1999). Penetrating the book-to market black box: The I+D effect. Journal of Business Finance \& Accounting april-may: 419-450.

Lev, B. y P. Zarowin (1999). The Boundaries of Financial Reporting and How to Extend Them. Journal of Accounting Research 37 (2): 353-385.

Lo, K. y T. Lys (2000). The Ohlson model: contribution to valuation theory, limitations, and empirical applications. Journal of Accounting, Auditing and Finance 15 (3): 337-367.

McCrae, M. y H. Nilsson (2001). The explanatory and predictive power of different specifications of the Ohlson (1995) valuation models. The European Accounting Review 10 (2): 315-341.

Morel, M. (2003). Endogenous Parameter Time Series Estimation of the Ohlson Model: Linear and Nonlinear Analysis. Journal of Business, Finance and Accounting 30 (9-10): 1341-1363.

Mui-Siang, P. y C. Yeow Lim (2007). The vaue relevance fo accounting variables and analysts' forecasts. The case of biotechnology firms. Review for Accounting and Finance 6 (3): 233-253.

Nguyen, P., S. Nivoix y M. Noma (2010). The valuation of R\&D expenditures in Japan. Accounting and Finance 50: 899-920.

Ohlson, J. (1995). Earnings, Book Values, and Dividends in Equity Valuation. Contemporary Accounting Research 11-2: 661-687.

(1999). On Transitory Earnings. Review of Accounting Studies 4 (3-4): 145-162.

(2001). Earnings, Book Values, Dividends in Equity Valuation: An Empirical Perspective. Contemporary Accounting Research 18: 107-120. 
Oswald, D. (2008). The determinants and value relevance of the choice of accounting for research and development expenditures in the United Kingdom. Journal of Business finance \& Accounting january-march 35 (1-2): 1-24.

Oswald, D. y P. Zarowin (2007). Capitalization of I+D and the informativeness of Stock Price. European Accounting Review 16 (4): 703-726.

Seybert, N. (2010). R\&D Capitalization and Reputation-Driven Real Earnings Management. The Accounting Review 85 (2): 671-693.

Shortridge, T. (2004). Market Valuation of Successful versus Non-successful I+D Efforts in the Pharmaceutical Industry. Journal of Business Finance and Accounting 31 (9-10): 1301-1325.

Sougiannis, T. (1994). The Accounting Based Valuation of Corporate I+D. The Accounting Review 69 (1): 44-68.

(2001). The Stock Market Valuation of Research and Development Expenditures. The Journal of Finance 56 (6): 2431-2459.

Sundaram, A.K., T.A. John y K. John (1996). An Empirical Analysis of Strategic Competition and Firm Values. The case of R\&D Competition. Journal of Financial Economics 40: 459-489.

White, H. (1980). A Heteroscedasticity Consistent Covariance Matrix Estimator and a Direct Test of Heroscedasticity. Econométrica 48: 817-818.

Wyatt, A. (2008). What financial and non-financial information on intangibles is value-relevant? A review of the evidence. Accounting and business Research 38 (3): 217-259.

Zhao, R. (2002). Relative Value Relevance of I+D Reporting: An International Comparison. Journal of International Financial Management and Accounting 13 (2): 153-174. 


\section{Anexo I}

\section{Cuadro 1.1}

Estadística descriptiva de las variables divulgadas $P_{t}, B_{t}, x_{t}^{a}, I D_{t} \mathbf{y} I D_{t-1}$ para la muestra completa y para las DIL I y II

\begin{tabular}{c|c|c|c|c|c} 
& $P_{t}$ & $B_{t}$ & $x_{t}^{a}$ & $I D_{t}$ & $I D_{t-1}$ \\
\hline Media & 1.6661 & 0.4653 & -0.1408 & 1.1250 & 0.4169 \\
\hline Mediana & 0.6475 & 0.3443 & 0.0173 & 0.0244 & 0.0234 \\
\hline Máximo & 240.2528 & 210.8504 & 11.2822 & 35.5172 & 35.5172 \\
\hline Mínimo & 0.0002 & 1.9415 & -43.4808 & 0.0000 & 0.0000 \\
\hline Desv. Est. & 11.0340 & 9.4972 & 1.9821 & 2.8830 & 2.9208 \\
\hline Asimetría & 18.9520 & 15.9695 & -17.7732 & 11.2866 & 8.9416 \\
\hline Curtosis & 385.4946 & 414.4489 & 383.8898 & 87.0587 & 87.3927 \\
\hline $\mathrm{N}^{\mathrm{o}}$ Observ. & 611 & 611 & 611 & 611 & 611 \\
\hline
\end{tabular}

Las cifras presentadas en esta tabla están expresadas en miles de dólares por acción.

\section{Cuadro 1.2}

Medidas resúmenes de las variables divulgadas $I D_{t}$ y $I D_{t-1}$ por años y para las DIL. I y II

\begin{tabular}{c|c|c|c|c|c}
\hline \multirow{2}{*}{ Año } & \multirow{2}{*}{$\mathbf{N}^{\mathbf{*}}$} & \multicolumn{2}{|c|}{$D_{t}$} & \multicolumn{2}{c}{$D_{t-1}$} \\
\cline { 3 - 6 } & & Media & Mediana & Media & Mediana \\
\hline 1995 & 37 & 2.4585 & 0.0175 & 4.0769 & 0.0162 \\
\hline 1996 & 39 & 3.1346 & 0.0239 & 4.8454 & 0.0212 \\
\hline 1997 & 52 & 2.0995 & 0.0186 & 2.4021 & 0.0202 \\
\hline 1998 & 51 & 1.8868 & 0.0228 & 2.1017 & 0.0256 \\
\hline 1999 & 50 & 3.8427 & 0.0204 & 1.9192 & 0.0190 \\
\hline 2000 & 40 & 4.6447 & 0.0246 & 4.7972 & 0.0242 \\
\hline 2001 & 44 & 2.5471 & 0.0276 & 2.4656 & 0.0236 \\
\hline 2002 & 44 & 4.9467 & 0.0360 & 4.5962 & 0.0330 \\
\hline 2003 & 47 & 0.8470 & 0.0258 & 0.9883 & 0.0280 \\
\hline
\end{tabular}

Las cifras presentadas en esta tabla están expresadas en miles de dólares por acción. 


\section{Anexo II}

\section{Cuadro 2.1}

Composición del coste de capital $\left(r_{i}\right)$ para las empresas de USA y del Reino Unido (RU) que forman nuestra muestra y para el periodo 1995-2004

\begin{tabular}{c|c|c}
\hline Año & $r_{U S A}$ & $r_{R U}$ \\
\hline 1995 & $0.0569+0.06$ & $0.0633+0.04$ \\
\hline 1996 & $0.0523+0.06$ & $0.0578+0.04$ \\
\hline 1997 & $0.0536+0.06$ & $0.065+0.04$ \\
\hline 1998 & $0.0485+0.06$ & $0.0681+0.04$ \\
\hline 1999 & $0.0478+0.06$ & $0.0504+0.04$ \\
\hline 2000 & $0.0585+0.04$ & $0.058+0.04$ \\
\hline 2001 & $0.0424+0.04$ & $0.0476+0.04$ \\
\hline 2002 & $0.0161+0.04$ & $0.0386+0.04$ \\
\hline 2003 & $0.0101+0.04$ & $0.0356+0.04$ \\
\hline 2004 & $0.0139+0.04$ & $0.0444+0.04$ \\
\hline
\end{tabular}

\section{Anexo III}

\section{Matrices de correlación correspondiente a las variables} que forman la función de valoración de la DILI

Matriz de correlación para el periodo 1995

\begin{tabular}{c|c|c|c|c}
\hline & $P_{t}$ & $B_{t}$ & $x_{t}^{a}$ & $I D_{t}$ \\
\hline$P_{t}$ & 1.0000 & 0.9600 & 0.9973 & 0.3473 \\
\hline$B_{t}$ & 0.9600 & 1.0000 & 0.9970 & 0.3495 \\
\hline$x_{t}^{a}$ & 0.9973 & 0.9970 & 1.0000 & 0.3516 \\
\hline$I D_{t}$ & 0.3473 & 0.3495 & 0.3516 & 1.0000 \\
\hline
\end{tabular}




\section{Matrices de correlación correspondiente a las variables que forman la función de valoración de la DILII}

Matriz de correlación para el periodo 1997

\begin{tabular}{c|c|c|c|c|c}
\hline & $P_{t}$ & $B_{t}$ & $x_{t}^{a}$ & $I D_{t}$ & $I D_{t-1}$ \\
\hline$P_{t}$ & 1.0000 & 0.6879 & 0.9424 & -0.1378 & -0.1384 \\
\hline$B_{t}$ & 0.6879 & 1.0000 & 0.5313 & -0.2520 & -0.2503 \\
\hline$x_{t}^{a}$ & 0.9424 & 0.5313 & 1.0000 & -0.0434 & -0.0449 \\
\hline$I D_{t}$ & -0.1378 & -0.2520 & -0.0434 & 1.0000 & 0.9995 \\
\hline$I D_{t-1}$ & -0.1384 & -0.2503 & -0.0449 & 0.9995 & 1.0000 \\
\hline
\end{tabular}

Matriz de correlación para el periodo 2003

\begin{tabular}{c|c|c|c|c|c}
\hline & $P_{t}$ & $B_{t}$ & $x_{t}^{a}$ & $I D_{t}$ & $I D_{t-1}$ \\
\hline$P_{t}$ & 1.0000 & 0.4726 & 0.9448 & -0.1664 & -0.1671 \\
\hline$B_{t}$ & 0.4726 & 1.0000 & 0.2917 & -0.3651 & -0.3671 \\
\hline$x_{t}^{a}$ & 0.9448 & 0.2917 & 1.0000 & -0.1205 & -0.1200 \\
\hline$I D_{t}$ & -0.1664 & -0.3651 & -0.1205 & 1.0000 & 0.9985 \\
\hline$I D_{t-1}$ & -0.1671 & -0.3671 & -0.1200 & 0.9985 & 1.0000 \\
\hline
\end{tabular}

